\title{
Striatal Dopamine Transporter Availability Associated with Polymorphisms in the Dopamine Transporter Gene SLC6A3
}

Elisabeth M. van de Giessen ${ }^{1,2}$, Maartje M.L. de Win ${ }^{3}$, Michael W.T. Tanck ${ }^{4}$, Wim van den Brink ${ }^{5}$, Frank Baas ${ }^{2}$, and Jan Booij ${ }^{1}$

${ }^{1}$ Department of Nuclear Medicine, Academic Medical Center, University of Amsterdam, Amsterdam, The Netherlands; ${ }^{2}$ Department of Neurogenetics, Academic Medical Center, University of Amsterdam, Amsterdam, The Netherlands; ${ }^{3}$ Department of Radiology, Academic Medical Center, University of Amsterdam, Amsterdam, The Netherlands; ${ }^{4}$ Department of Clinical Epidemiology, Biostatistics and Bioinformatics, Academic Medical Center, University of Amsterdam, Amsterdam, The Netherlands; and ${ }^{5}$ Department of Psychiatry, Academic Medical Center, University of Amsterdam, Amsterdam, The Netherlands

Polymorphisms in the dopamine transporter (DAT) gene SLC6A3 are associated with human striatal DAT expression, but the exact effects on DAT expression are not clear. A variable number of tandem repeats (VNTR) in the $3^{\prime}$ untranslated region of the DAT gene was previously investigated in relation to striatal DAT availability, but the results were inconclusive. Other polymorphisms in the DAT gene were not extensively studied. Therefore, we investigated whether polymorphisms in both $3^{\prime}$ and $5^{\prime}$ ends of the DAT gene show association with in vivo striatal DAT expression. Methods: The subjects were an ethnically homogeneous group of 79 healthy young adults. Striatal DAT availability was measured with ${ }^{123} \mathrm{I}-(2-\beta$-carbomethoxy-3- $\beta$ (4-iodophenyl)-tropane) (123I- $\beta$-CIT) SPECT. The 40 -base-pair VNTR in the $3^{\prime}$ untranslated region of the DAT gene and the 2 single nucleotide polymorphisms (SNPs) rs2652511 and rs2937639 in the 5' end of the DAT gene were genotyped. Multiple-regression analysis was performed for each of the 3 polymorphisms. Analysis of the combination of the polymorphisms (haplotype analysis) was conducted for the triad rs2652511-rs2937639-VNTR. Results: For the VNTR, the 9-repeat (9R) allele was associated with significantly higher striatal DAT expression than was the 10-repeat (10R) allele $(P=0.002)$. Subanalysis suggested a dominant effect for the 9R allele. Neither SNP rs2652511 nor SNP rs2937639 was associated with striatal DAT availability. The haplotype T-A-9R (rs2652511-rs2937639-VNTR) was significantly more associated with higher striatal DAT expression than were the other haplotypes $(P=0.009)$. Conclusion: The DAT VNTR 9R carriers have higher striatal DAT availability than do 10R homozygotes. This finding replicates former studies that included healthy subjects and also used ${ }^{123}$ - $\beta$-CIT SPECT. Our haplotype analysis identified a subgroup of $9 R$ carriers, the T-A-9R, which appears to be mainly responsible for the association with higher striatal DAT availability. Thus, a combination of polymorphisms in both the $3^{\prime}$ and the $5^{\prime}$ ends of the DAT gene is associated with in vivo striatal DAT expression. This finding in healthy subjects

Received Apr. 17, 2008; revision accepted Sep. 22, 2008.

For correspondence or reprints contact: Elisabeth M. van de Giessen, Department of Nuclear Medicine, Academic Medical Center, P.O. Box 22660, 1100 DD Amsterdam, The Netherlands.

E-mail: e.m.vandegiessen@amc.uva.nl

COPYRIGHT ๑ 2009 by the Society of Nuclear Medicine, Inc. may contribute to research on DAT availability and genotype in neuropsychiatric disorders.

Key Words: dopamine transporter (DAT); SLC6A3; genotype; striatum; ${ }^{123} \mathrm{I}-\beta-\mathrm{CIT}$ SPECT

J Nucl Med 2009; 50:45-52

DOI: 10.2967/jnumed.108.053652

$\mathbf{P}$ olymorphisms in the gene SLC6A3 for the dopamine transporter (DAT) have been associated with DAT availability in the human brain. This finding suggests an effect of these polymorphisms, or of neighboring polymorphisms that are inherited together with them, on gene expression. A variable number of tandem repeats (VNTR) in the 3' untranslated region of the gene is the most extensively studied polymorphism in in vitro and in vivo studies for DAT gene expression. This VNTR has been associated with several neuropsychiatric disorders, in particular attention deficit hyperactivity disorder (ADHD) (1). Two other studies suggested that combinations of polymorphisms (haplotypes) in the $5^{\prime}$ end and throughout the gene affect DAT expression $(2,3)$. However, results are inconclusive about the exact effects of the polymorphisms on DAT expression.

The VNTR in the $3^{\prime}$ untranslated region has 2 alleles that are most common: a 9-repeat (9R) and 10-repeat (10R) of a 40-base-pair (bp) sequence. Less common alleles range from 3 to 11 repeats. In vitro studies suggested a significant effect of the VNTR on gene expression, but conflicting results exist regarding the effects of the $9 \mathrm{R}$ and $10 \mathrm{R}$ alleles (3-7). Several in vivo SPECT studies measured gene expression by DAT availability. Two studies in healthy subjects reported significantly lower striatal DAT binding for 10R homozygotes $(8,9)$. Studies that also included subjects with neuropsychiatric disorders showed different results: 2 showed increased striatal DAT availability for 10R homo- 
zygotes $(10,11)$, and 3 found no association (12-14). Although differences in population, radioligand, and sample size can partially explain the inconsistent results, data on the specific effect of the VNTR polymorphism on striatal DAT binding in humans remain inconclusive.

It is likely that polymorphisms in DAT gene regions other than that around the VNTR also influence gene expression. Analysis of high-linkage disequilibrium regions across the gene reveals the presence of 2 blocks defining the $5^{\prime}$ (promotor to intron 6) and $3^{\prime}$ (exon 9 to exon 15) regions of the gene (15). Drgon et al. (2) screened the $5^{\prime}$ end of SLC6A3 for polymorphisms and identified a core combination of 2 single nucleotide polymorphisms (SNPs; T841C/rs2652511/C-839T and A+1821G/rs2937639/G+1736A) that captures most information of this region. They found that ventral striatal DAT availability measured with ${ }^{11} \mathrm{C}$ cocaine PET was significantly higher for the $\mathrm{C}-\mathrm{G}$ combination (rs2652511-rs2937639) than for the T-A combination in 15 European American subjects (9 controls and 6 patients with ADHD). The $\mathrm{C}-\mathrm{G}$ combination also showed higher levels of striatal DATs in postmortem brains, suggesting higher striatal DAT expression. There is conflicting evidence about the effect of polymorphism blocks in this gene, though. Greenwood and Kelsoe (3) also identified a combination of polymorphisms at the $5^{\prime}$ end of the DAT gene. One of the SNPs in the combinations identified by Greenwood et al. and Drgon et al. is the same $(\mathrm{A}+1821 \mathrm{G} /$ rs2937639/G+1736A). However, the overlapping combination of the SNP documented by Greenwood et al. showed an opposite effect of lower expression of DAT in SN4741 cell lines that contain this polymorphism combination.

In view of these conflicting results, we set out to investigate the relationship between the level of human striatal DAT expression and polymorphisms in both $5^{\prime}$ and $3^{\prime}$ ends, that is, the 2 high-linkage disequilibrium blocks, of the DAT gene in a relatively large, ethnically homogeneous sample of healthy subjects.

\section{MATERIALS AND METHODS}

\section{Subjects}

A total of 81 Caucasian healthy young adults were recruited. They were participants in the prospective cohort study of The Netherlands XTC Toxicity (NeXT) study. In a special design paper on this study, a detailed description is given of recruitment strategies, exclusion criteria, and other methods (16). Subjects were selected on the basis of their high probability of starting to use ecstasy. They were actively recruited using a combination of targeted site-sampling at locations such as dance events, discotheques, youth fairs, universities, colleges, and parks; advertisement through a Web site on the project and an Internet campaign; and snowball sampling referrals. The main criteria for inclusion were intent to use ecstasy for the first time in the near future (3-5 points on a 5-point scale: 1, certainly not; 2, probably not; 3 , undecided; 4, probably yes; and 5, certainly yes). All subjects were ecstasy-naïve at the time of examination for the current study. Exclusion criteria included presence of a severe medical or neuropsychiatric disorder (e.g., depression, psychosis, or parkinsonism), use of psychotropic medications, pregnancy, and use of intravenous drugs. The subjects had to abstain from the use of psychoactive substances for at least $2 \mathrm{wk}$ before examinations and from alcohol for at least $1 \mathrm{wk}$ before examinations. Abstinence was checked by urine testing (enzyme-multiplied immunoassay for amphetamines, 3,4-methylenedioxymethamphetamine, opioids, cocaine, benzodiazepine, cannabis, and alcohol).

The study was approved by the local medical ethics committee, and all subjects gave written informed consent. The subjects received remuneration for their participation in the NeXT study.

\section{SPECT}

Acquisition. A SPECT scan was performed with the radioligand ${ }^{123} \mathrm{I}$-(2- $\beta$-carbomethoxy-3- $\beta$ (4-iodophenyl)-tropane) ( ${ }^{123} \mathrm{I}-\beta$-CIT), which has a high affinity for the DAT. Radiosynthesis of ${ }^{123} \mathrm{I}-\beta$ CIT and SPECT camera and image acquisitions was described previously (17). SPECT images were acquired $24 \mathrm{~h}$ after injection of the radioligand, when stable specific binding uptake to striatal DATs was expected to be reached (18).

Postprocessing. Attenuation correction of all images was performed as described earlier (19). For quantification, a regionof-interest (ROI) analysis was performed. Standardized templates of 2-dimensional ROIs were drawn with the help of high-resolution MR images and a brain atlas. ROIs for the whole striatum, caudate nucleus, putamen, and occipital cortex were used. The ROIs were positioned on the 3 consecutive axial slices with highest striatal activity (Fig. 1). Mean striatal, mean caudate nucleus, mean putamen, and mean occipital binding densities were averaged from right and left ROIs on the 3 slices. Activity in the occipital cortex was assumed to represent nondisplaceable activity (nonspecific binding and free radioactivity). Ratios of specific binding to nonspecific binding were calculated as the nondisplaceable binding potential $\left(\mathrm{BP}_{\mathrm{ND}}\right)=$ (activity in ROI activity in occipital cortex)/activity in occipital cortex $(18,20)$.
FIGURE 1. ROls for quantification analysis of ${ }^{123} \mid-\beta-$ CIT scan are shown in the same image, with different shades of intensity. To visualize low binding in occipital cortex (right), upper threshold was set at approximately $13 \%$ of maximum of study.

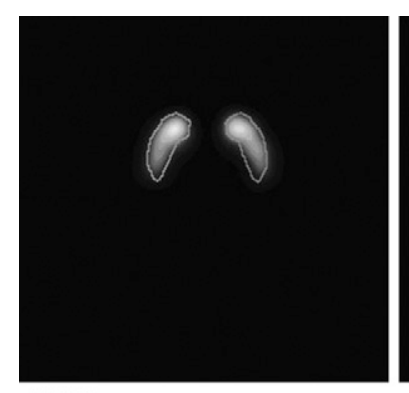

Striatum

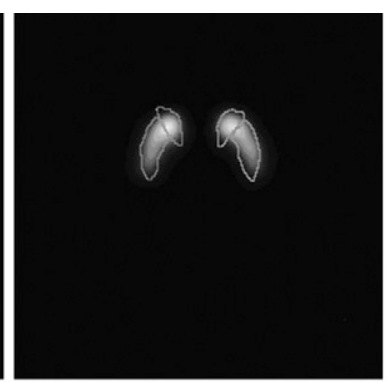

Caudate nucleus and putamen

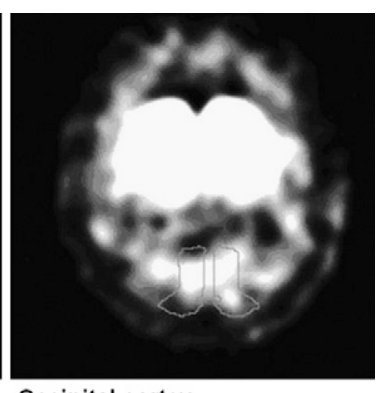

Occipital cortex 


\section{Genotyping}

The DNA of the subjects was extracted from whole-blood samples using standard protocols. We genotyped the 40-bp VNTR in the 3' untranslated region of the DAT gene SLC6A3 and the 2 SNPs rs2652511 and rs2937639 in the $5^{\prime}$ end (Fig. 2), which were identified as the core polymorphism combination by Drgon et al. (2). With these polymorphisms, we covered the 2 haplotype blocks identified by Greenwood et al. (15). The VNTR and the SNPs with at least $100 \mathrm{bp}$ of flanking intronic sequences were amplified from the DNA by polymerase chain reaction (PCR). For the VNTR, we used the primers described by Vandenbergh et al. (21). For the SNPs, primers were designed with Primer3, version 0.4.0 (22) (rs2652511: forward primer, GCT GGA ATG GCT GGA GAG, and reverse primer, CGC CTA AGA AAA CCA TTT CC; rs2937639: forward primer, AAA TAA CTT AGC CGG TGC TG, and reverse primer, GGC CTC AAG ACA GAC ACT CT). The amplification reaction followed a standard protocol. PCR products for VNTR analysis were seperated by gel electrophoresis in $3 \%$ agarose gel stained with ethidium bromide. PCR products for rs2652511 and rs2937639 were directly sequenced, using a Prism BigDye Terminator Cycle Sequencing Ready Reaction kit (Applied Biosystems), and run on a genetic analyzer (3730; Applied Biosystems). Sequence data were analyzed using software (CodonCode Aligner, version 1.6.3; CodonCode Corp.).

\section{Statistical Analysis}

The 3 markers, rs2652511, rs2937639, and the VNTR, were tested for Hardy-Weinberg equilibrium. Multiple-regression analysis was used with ${ }^{123}$ I- $\beta$-CIT binding to DATs as the dependent variable and the different genotype markers as the independent variables. These analyses were corrected for age and sex, because these variables may influence in vivo DAT binding in humans $(23,24)$. The following 4 multiple-regression models were tested for each genetic marker: genotype, comparing homozygotes a-a, heterozygotes a-b, and homozygotes $\mathrm{b}-\mathrm{b}$ as 3 independent groups; allele dose effect, testing for a linear relationship for homozygotes $a-a$, heterozygotes $a-b$, and homozygotes b-b; dominant, comparing allele a carriers with homozygotes $b-b$; and recessive, comparing homozygotes $a-a$ with allele $b$ carriers.

Because the included subjects were selected on a basis of a relatively high probability of starting to use ecstasy, substanceabuse parameters (nicotine, alcohol, cannabis, amphetamines, and cocaine) were screened for potential confounder effects. Potential confounders were defined as variables that may be related to the dependent-variable DAT availability at the $P<0.20$ level of significance (25). In additional multiple-regression analysis, the observed relationships were adjusted for the effect of these potential confounders. SPSS software (version 12.0.1; SPSS Inc.) was used for regression analyses.

The effect of the possible allele combinations (haplotypes) for rs2652511-rs2937639-VNTR on DAT binding level was also

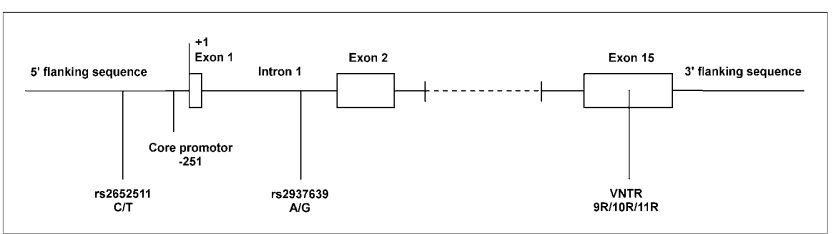

FIGURE 2. $5^{\prime}$ and $3^{\prime}$ ends of DAT gene. analyzed. Haplotype analysis according to the method of Tanck et al. (26) was used. In short, haplotype effects and haplotype frequencies were jointly estimated using an expectation-maximization algorithm in which individual haplotypes were handled as missing data. In the first expectation (E) step, the initial probabilities were calculated using Bayes' theorem and estimated haplotype frequencies. In the following $\mathrm{E}$ steps, the posterior probabilities of haplotype pairs compatible with the genotype of an individual were calculated on the basis of the phenotype of the individual subject. In the maximization (M) steps, the haplotype effects were estimated using a weighted linear-regression model, in which the posterior probabilities functioned as weights. The $\mathrm{E}$ and $\mathrm{M}$ steps were alternated until they converged. The haplotype analysis compared the DAT availability of all haplotypes in 1 model. A post hoc analysis was used for pairwise comparisons of the DAT availability of the individual haplotypes. The haplotype analyses were corrected for age and sex.

Bonferroni adjustment was performed for all analyses, to correct for multiple testing. As analyses were performed for the 3 polymorphisms separately, with an additional haplotype analysis, the significance level after Bonferroni adjustment was corrected to 0.012 . For the post hoc analyses for pairwise comparison of haplotypes, the significance level was set at 0.005 , as there were 10 pairwise comparisons.

The genetic power calculator confirmed that this study had greater than $90 \%$ power to detect a DAT level change of $15 \%$, with a marker with a minor allele frequency of 0.1 (27).

\section{RESULTS}

VNTR and both SNPs were in Hardy-Weinberg equilibrium for the 81 recruited subjects. Allele frequencies for VNTR were 0.226 for $9 \mathrm{R}, 0.750$ for $10 \mathrm{R}$, and 0.024 for 11R; the frequency for rs 2652511 was 0.393 for $\mathrm{T}$, and for rs 2937639 it was 0.399 for A. Two subjects had the rare VNTR genotype 10R/11R and were excluded, leaving 79 subjects for the regression and haplotype analyses. Sample characteristics of the 79 subjects are shown in Table 1. The genotyping failed for 2 DNA samples for rs 2652511 , for 2 DNA samples for rs2937639, and for 2 DNA samples for the VNTR and left 74 subjects with complete genotype information on the 3 polymorphisms.

\section{VNTR, rs2652511, and rs2937639 Analysis}

Distribution of VNTR genotype by striatal DAT availability is shown in Figure 3. Multiple-regression analysis showed a significant association between striatal DAT availability and VNTR genotype (Table 2). This association was strongest in the dominant model that compared 9R carriers with 10R homozygotes. The 9R allele-recessive model showed no significant associations. The regression coefficients (B) in Table 2 indicate that 9R genotypes are associated with higher striatal DAT availability than are 10R genotypes. The proportion of variance explained by VNTR genotype was highest in the 9R-dominant model $\left(R^{2}=0.178\right)$. The analysis of the subregions of the striatum, caudate nucleus, and putamen showed similar results, with the strongest associations again in the 9R-dominant model and no association in the 9R-recessive model (Table 2). All 


\begin{tabular}{|c|c|}
\hline Characteristic & Data \\
\hline Number of subjects $(n)$ & 79 \\
\hline Male/female $(n)$ & $33 / 46$ \\
\hline \multicolumn{2}{|l|}{ Age (y) } \\
\hline Mean $\pm S D$ & $22.0 \pm 3.5$ \\
\hline Range & $18-35$ \\
\hline \multicolumn{2}{|l|}{$\mathrm{BP}_{\mathrm{ND}}($ mean $\pm \mathrm{SD})$} \\
\hline Striatum & $11.3 \pm 2.7$ \\
\hline Caudate nucleus & $12.2 \pm 2.9$ \\
\hline Putamen & $10.3 \pm 2.5$ \\
\hline \multicolumn{2}{|l|}{ VNTR (n) } \\
\hline 9R/9R & 5 \\
\hline 9R/10R & 27 \\
\hline 10R/10R & 45 \\
\hline \multicolumn{2}{|l|}{ rs2652511 (n) } \\
\hline $\mathrm{CC}$ & 29 \\
\hline CT & 35 \\
\hline$\pi$ & 13 \\
\hline \multicolumn{2}{|l|}{ rs2937639 (n) } \\
\hline GG & 28 \\
\hline AG & 36 \\
\hline AA & 13 \\
\hline \multicolumn{2}{|l|}{ Substance use (mean \pm SD) } \\
\hline Alcohol (units/wk) & $8.7 \pm 9.2$ \\
\hline Tobacco (cigarettes/wk) & $32.5 \pm 55.6$ \\
\hline Cannabis (joints last year) & $37.1 \pm 89.2$ \\
\hline Amphetamine (times last year) & $0.1 \pm 0.7$ \\
\hline Cocaine (times last year) & $0.4 \pm 1.5$ \\
\hline
\end{tabular}

significant findings remained significant after Bonferroni adjustment.

For the SNPs rs2652511 and rs2937639, no association was found with DAT availability in the striatum, caudate nucleus, or putamen.

In the allele dose-effect model and recessive model of the multiple-regression analysis for the VNTR, sex showed a significant association with striatal DAT availability: $\mathrm{B}=$ 1.3 (95\% confidence interval $[\mathrm{CI}], 0.9-2.4), P=0.036$, and $\mathrm{B}=1.3(95 \% \mathrm{CI}, 0.8-2.5), P=0.037$, respectively. In the other models, sex was close to significance $(0.10>P>$ $0.05)$

The only identified potential confounder of the substance-abuse parameters was number of cigarettes per week $(P=0.11)$. When number of cigarettes per week was added to multiple-regression analysis, only small effects on results were observed: genotype model: $P=0.098$ and $\mathrm{B}=2.0$ (95\% CI, -0.4 to 4.3 ) for $9 \mathrm{R} / 9 \mathrm{R}$ vs. $10 \mathrm{R} / 10 \mathrm{R}$, and $P=$ 0.006 and $\mathrm{B}=1.7$ (95\% CI, 0.5-3.0) for $9 \mathrm{R} / 10 \mathrm{R}$ vs. $10 \mathrm{R} /$ 10R; allele-dose-effect model: $P=0.005$ and $\mathrm{B}=-1.4$ (95\% CI, -2.3 to -0.4$)$; 9R-dominant model: $P=0.003$ and $\mathrm{B}=-1.8(95 \% \mathrm{CI},-2.9$ to -0.6$)$; and 9R-recessive model: $P=0.266$ and $\mathrm{B}=-1.4(95 \% \mathrm{CI},-3.8$ to 1.1$)$.

\section{Haplotype Analysis}

The alleles of the 2 SNPs (rs2652511 and rs2937639) appeared to be inherited together in 2 fixed combinations (either $\mathrm{T}-\mathrm{A}$ or $\mathrm{C}-\mathrm{G})$, except for 1 subject $(\mathrm{C}-\mathrm{A})$ showing

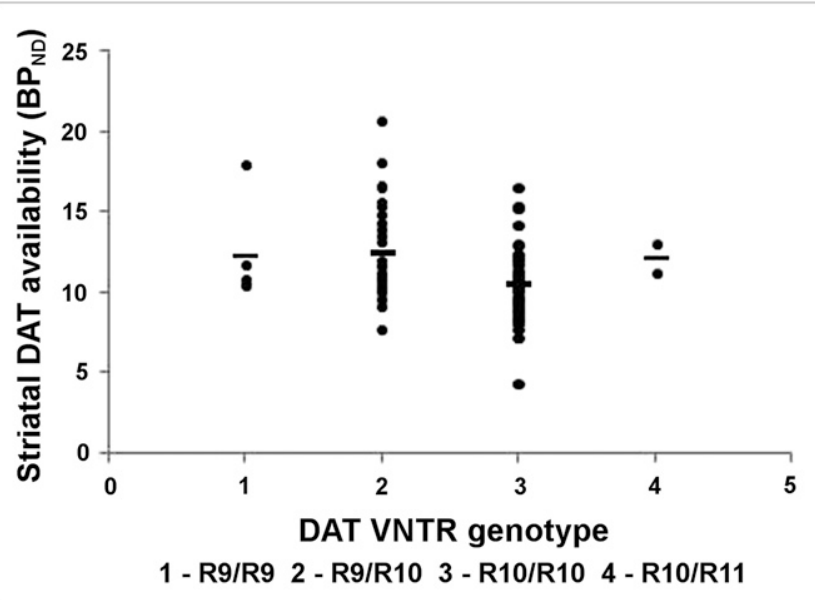

FIGURE 3. Striatal DAT availability $\left(\mathrm{BP}_{\mathrm{ND}}\right)$ of each subject by DAT genotype. Mean $\mathrm{BP}_{\mathrm{ND}}$ is indicated by vertical mark for each genotype.

high linkage disequilibrium $\left(\mathrm{D}^{\prime}=0.97\right)$. The overall $P$ value of the haplotype effects model was $4.3 \times 10^{-5}$. One haplotype (rs2652511-rs2937639-VNTR: T-A-9R) had significantly different striatal DAT availability, compared with all other haplotypes in the model $\left(R^{2}=0.37 ; \mathrm{B}=3.2\right.$ [95\% CI, 0.8-5.5], $P=0.009$; Table 3) and compared with each individual haplotype in the post hoc analyses (Table 3). The significant associations remained significant after Bonferroni adjustment, except for the pairwise association between haplotype T-A-9R and the rare haplotype C-A10R. The positive regression coefficient $B$ showed that the striatal DAT availability of T-A-9R is higher than that of the other haplotypes. No other haplotypes differed significantly from the group or from each other. Figure 4 shows the effect of each haplotype (regression coefficient B and $95 \%$ CI) on striatal DAT availability according to the haplotype analysis with correction for age and sex.

\section{DISCUSSION}

This study showed that DAT genotype is associated with in vivo striatal DAT availability in our study population of young Caucasian adults. First, the 40-bp repeat VNTR in the $3^{\prime}$ untranslated region demonstrated an effect: subjects with 9R had higher striatal DAT availability than did subjects with $10 \mathrm{R}$. The highly significant association in the 9R-dominant model and the lack of a significant association in the 9R-recessive model suggest that the 9R allele displays a dominant effect. Analysis of the striatal subregions caudate nucleus and putamen showed that both regions contribute to this effect.

Interestingly, the 2 other studies with healthy subjects that used ${ }^{123}$ I- $\beta$-CIT as a ligand $(8,9)$ demonstrated the same effect of the VNTR: 9R carriers have higher striatal DAT availability than do 10R homozygotes. Jacobsen et al. found a significant difference in striatal DAT binding of $13.4 \%$ in 30 healthy subjects (8). Van Dyck et al. found a 
TABLE 2. Regression Analysis for DAT VNTR and Striatal DAT Availability

\begin{tabular}{|c|c|c|c|c|c|c|}
\hline \multirow[b]{3}{*}{ Model } & \multicolumn{6}{|c|}{ DAT availability } \\
\hline & \multicolumn{2}{|c|}{ Striatum } & \multicolumn{2}{|c|}{ Caudate } & \multicolumn{2}{|c|}{ Putamen } \\
\hline & $P$ & $\mathrm{~B}(95 \% \mathrm{Cl})^{*}$ & $P$ & $\mathrm{~B}(95 \% \mathrm{Cl})^{*}$ & $P$ & $\mathrm{~B}(95 \% \mathrm{Cl})^{*}$ \\
\hline \multicolumn{7}{|l|}{ Genotype } \\
\hline $10 \mathrm{R} / 10 \mathrm{R}$ vs. $9 \mathrm{R} / 9 \mathrm{R}$ & 0.099 & $2.0(-0.4-4.3)$ & 0.063 & $2.5(-0.1-5.1)$ & 0.014 & $1.6(-0.6-3.8)$ \\
\hline $10 R / 10 R$ vs. $9 R / 10 R$ & 0.003 & $1.9(0.6-3.1)$ & 0.004 & $2.0(0.7-3.3)$ & 0.003 & $1.8(0.6-2.9)$ \\
\hline Allele dose effect & 0.003 & $-1.4(-2.3-0.5)$ & 0.002 & $-1.6(-2.6-0.6)$ & 0.004 & $-1.3(-2.1-0.4)$ \\
\hline 9R dominant & 0.002 & $-1.9(-3.0-0.7)$ & 0.002 & $-2.1(-3.3-0.8)$ & 0.002 & $-1.7(-2.8-0.7)$ \\
\hline 9R recessive & 0.288 & $-1.3(-3.8-1.1)$ & 0.200 & $-1.7(-4.4-0.9)$ & 0.386 & $-1.0(-3.3-1.3)$ \\
\hline
\end{tabular}

${ }^{*}$ Regression coefficient $\mathrm{B}$.

All regression analyses are corrected for age and sex.

striatal DAT decrease in 10R homozygotes of $8.9 \%$ in a large sample of 96 healthy European Americans, who were extensively screened for neuropsychiatric confounders (9). It seems that the findings of these authors are confirmed with this study. However, Martinez et al. (12) did not find an effect of DAT genotype on striatal DAT availability measured with ${ }^{123}$ I- $\beta$-CIT SPECT in their group of 21 healthy controls and 22 subjects with schizophrenia. Lynch et al. (14) also did not find this association in their cohort of 66 healthy controls and 100 patients with Parkinson disease, but they measured striatal DAT availability with ${ }^{99} \mathrm{Tc}-2-[[2-[[[3-(4-$ chlorophenyl)-8-methyl-8-azabicyclo[3,2,1]oct-2-yl]methyl](2mercaptoethyl)amino]ethyl]amino]ethanethiolato(3-)- $N 2$, $N 2^{\prime}, S 2, S 2^{\prime}$ ] oxo-[1R-(exo-exo)] ( $\left.{ }^{99} \mathrm{Tc}-\mathrm{TRODAT}-1\right)$. Compared with ${ }^{99} \mathrm{Tc}-\mathrm{TRODAT}-1,{ }^{123} \mathrm{I}-\beta$-CIT SPECT has the advantage that at $24 \mathrm{~h}$ after injection, the in vivo binding of ${ }^{123} \mathrm{I}-\beta$-CIT to striatal DATs is in equilibrium $(18,19)$, and the binding ratios are much higher. All the other studies that demonstrated different effects of the VNTR on striatal DAT availability $(10,11,13)$ included subjects with neuropsychiatric disorders and did not analyze the healthy controls separately. In a combined cohort of 14 abstinent alcoholics and 11 controls, Heinz et al. (10) demonstrated an inverse effect of $22 \%$ increased DAT availability in the putamen, but not in the caudate nucleus, for 10R homozygotes as measured with ${ }^{123}$ I- $\beta$-CIT SPECT. These authors used the free binding potential (specific tracer binding in brain/free ${ }^{123}$ I- $\beta$-CIT concentration in plasma) as an outcome measure, whereas the other studies used $\mathrm{BP}_{\mathrm{ND}}$. Cheon et al. (11) also found significantly increased striatal DAT binding in 10R homozygotes in 11 children with ADHD, using ${ }^{123}$ I-labeled $N$-(3iodopropen-2-yl)-2 $\beta$-carbomethoxy-3 $\beta$-(chlorophenyl)tropane as a radioligand for the DAT. A recent study with only patients with schizophrenia $(n=61)$ found no associations between the VNTR and DAT availability measured with ${ }^{123}$ I-FP-CIT SPECT (13). The methodologic differences in subject population, sample size, radioligand, and outcome measures of the studies on DAT availability and DAT genotype could mainly explain the variable findings. Although a definite conclusion on the effect of the VNTR on DAT

\section{TABLE 3. Haplotype analysis and Post Hoc Pairwise Comparison of Haplotypes}

\begin{tabular}{|c|c|c|c|c|c|c|c|}
\hline \multirow[b]{2}{*}{ Data } & \multirow[b]{2}{*}{ Frequency (\%) } & \multirow[b]{2}{*}{$\mathrm{B}(95 \% \mathrm{Cl})^{\star}$} & \multirow[b]{2}{*}{$P$} & \multicolumn{4}{|c|}{$P$ value pairwise comparison of haplotype } \\
\hline & & & & C-G-9R & C-G-10R & T-A-10R & C-A-10R \\
\hline \multicolumn{8}{|l|}{ Haplotype $^{\dagger}$} \\
\hline T-A-9R & 9.6 & $3.2(0.8-5.5)$ & 0.009 & $2.75 \times 10^{-4}$ & $2.48 \times 10^{-5}$ & $5.52 \times 10^{-6}$ & $0.015^{\ddagger}$ \\
\hline C-G-9R & 14.7 & $-0.0(-2.1-2.1)$ & 0.974 & & 0.845 & 0.711 & 0.236 \\
\hline C-G-10R & 46.1 & $0.1(-1.9-2.1)$ & 0.935 & & & 0.433 & 0.216 \\
\hline T-A-10R & 28.9 & $-0.3(-2.3-1.8)$ & 0.803 & & & & 0.268 \\
\hline C-A-10R & 0.7 & $-3.0(-8.0-2.1)$ & 0.257 & & & & \\
\hline \multicolumn{8}{|l|}{ Covariate } \\
\hline Age & & $0.1(-0.1-0.2)$ & 0.320 & & & & \\
\hline Sex & & $1.0(-0.1-2.1)$ & 0.091 & & & & \\
\hline \multicolumn{8}{|c|}{$\begin{array}{l}{ }^{*} \text { Regression coefficient B. } \\
{ }^{\dagger} \text { Haplotypes are shown in sequence rs2652511-rs2937639-VNTR. } \\
{ }^{\dagger} \text { Not significant after Bonferroni adjustment for multiple testing. } \\
\text { Analyses are corrected for age and sex. } n=74 \text {. }\end{array}$} \\
\hline
\end{tabular}




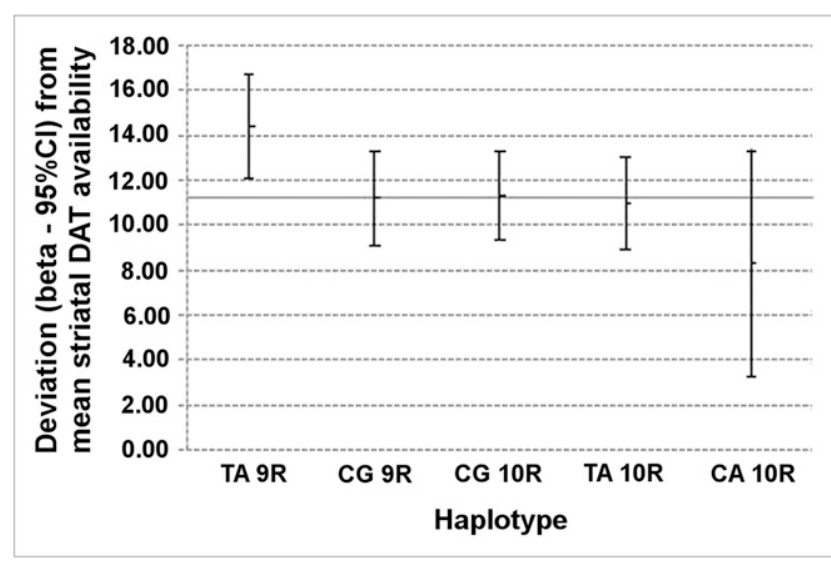

FIGURE 4. Haplotype analysis for rs2652511-rs2937639VNTR corrected for age and sex.

expression is not yet possible, the studies that included relatively large samples of healthy subjects and that used ${ }^{123} \mathrm{I}-\beta$-CIT as a radiotracer seem to indicate that $9 \mathrm{R}$ carriers have higher striatal DAT availability than do 10R homozygotes in healthy adults.

An additional result of this study is that haplotype T-A9R (rs2652511-rs2937639-VNTR) is associated with significantly higher striatal DAT availability than the other haplotypes. The other 9R haplotype (C-G-9R) does not deviate from the other haplotypes, nor does any $10 \mathrm{R}$ haplotype (Table 3 ). This would implicate that the finding that $9 \mathrm{R}$ carriers have higher in vivo striatal DAT availability than $10 \mathrm{R}$ homozygotes is mainly due to the subgroup of T-A-9R carriers. Although the 9R carriers would explain up to $18 \%$ of the variance in striatal DAT availability, the T-A9R carriers explain up to $37 \%$. These percentages cannot simply be compared, though, as they arose from different types of analyses.

The association with haplotype T-A-9R may also implicate that a combination of polymorphisms in both $3^{\prime}$ and $5^{\prime}$ ends, that is, both haplotype blocks of the DAT gene (15), can affect DAT expression. rs2652511 is located in the 5' flanking sequence before the promoter of the DAT gene; rs2937639 lies in intron 1 of the DAT gene. Both SNPs do not cause a change in the amino acid sequence of the protein. Because the 2 SNPs mainly inherited as fixed allele combinations, that is, were in high linkage disequilibrium, it is possible that another polymorphism in this region would be responsible for the identified effect, possibly in the promoter region. Why this effect would be expressed only in combination with the $9 \mathrm{R}$ allele is not clear. The VNTR is located in the $3^{\prime}$ untranslated region of the DAT gene; thus, it does not change the amino acid sequence of the protein. The mechanism by which the VNTR could influence DAT expression has not yet been explained.

The original finding of Drgon et al. (2), that for rs2652511-rs2937639 the C-G haplotype had higher striatal DAT availability, could not be replicated. However, that study differed from our study in that it had a small sample size $(n=15)$, including 6 patients with ADHD, and that striatal DAT availability was measured with ${ }^{11} \mathrm{C}$-cocaine PET.

It is interesting that both the multiple-regression analyses on the individual polymorphisms and the haplotype analysis show a tendency toward a sex effect on striatal DAT availability. According to our data, women would have a trend toward higher striatal DAT availability. Several studies have reported a similar effect of sex on DAT, with females showing greater DAT uptake than males $(24,28)$. However, other studies could not confirm this association (29). We did not find any association between striatal DAT availability and age, an established factor of influence (24). Probably because of the narrow age range of the subjects, the confounding effect of age was largely reduced in this study.

Because the young adult subjects of the present study were participants in the NeXT study, they were selected on the basis of their relatively high probability of starting to use ecstasy. This selection poses a limitation to the external validity of the study, because the study subject population has higher levels of substance use than does the general population of the same age. Because substance abuse and addictive behaviors are associated with a dysregulated dopaminergic neurotransmission, this may have affected DAT availability. However, substance-abuse parameters appeared not to affect the striatal DAT availability in this study significantly. This finding can be explained as follows. First, in the present study, substance use in the study population was rather limited (8.7 drinks/wk, 32.5 cigarettes/wk, and 0.7 joints/wk), and only a few participants ever used amphetamines $(n=1)$ or cocaine $(n=5)$. None of the subjects met the criteria for drug abuse. Effects of amphetamine use on striatal ${ }^{123} \mathrm{I}$ - $\beta$-CIT binding ratios have been described in individuals with a history of combined ecstasy and amphetamine use (30). However, in that study, the participants had used amphetamines approximately 48 times in the past year. More subjects used cannabis $(n=$ 58), but no effect of cannabis use on striatal DAT availability is expected (31). Second, the participants were instructed not to use drugs of abuse, including cocaine, in the $2 \mathrm{wk}$ before they were scanned, and urine tests on drugs of abuse confirmed abstinence. Although elevated striatal DATs during acute cocaine abstinence as measured by ${ }^{123} \mathrm{I}-$ $\beta$-CIT SPECT have been described (32), effects up to $2 \mathrm{wk}$ after the last dose of cocaine have not been described. Interestingly, the only identified potential confounder of the substance-abuse parameters was number of cigarettes per week. However, this effect was not significant $(P=0.11)$ in this study. Previous studies using ${ }^{99} \mathrm{Tc}-\mathrm{TRODAT}$ pointed at an association between smoking and the expression of striatal DATs (33), although other larger studies using ${ }^{123} \mathrm{I}-$ $\beta$-CIT as a radiotracer did not find such an association (28). In addition, subjects with serious mental or physical disorders and subjects using psychotropic medications were excluded from the study. Depression scores (on Beck 
Depression Inventory), Impulsiveness scores (on Barratt Impulsiveness Scale), and Sensation-Seeking scores (on a Dutch adaptation of Sensation-Seeking Scale) were all in the reference range (i.e., in the range of healthy populations of the same age range) (34). Overall, we cannot rule out any effect of substance abuse on this study, but we believe that this effect would be small and that the results of this study are valuable for the general population as well.

Our data support previous findings of an association between higher striatal DAT availability and 9R carriers for the DAT VNTR in healthy subjects. More specifically, a subgroup of $9 \mathrm{R}$ carriers, the T-A-9R, seems to be responsible for this association. This finding in healthy subjects could assist future research on the relationship between neuropsychiatric disorders and striatal DAT availability and genotype. For example, ADHD has been associated with the $10 \mathrm{R}$ allele (1), which would suggest a lower striatal DAT availability for patients with ADHD. However, there are no conclusive results yet on the level of striatal DAT availability in patients with ADHD (35). Another example is Parkinson disease, which has an established association with decreased striatal DAT availability. One would expect that the T-A-9R haplotype could be a protective factor. There are no conclusive results yet for the effect of the DAT VNTR as a risk factor for Parkinson disease. However, Kelada et al. identified a haplotype corresponding with the T-A-9R haplotype that had a tendency of being a risk factor instead of a protective factor for Parkinson disease, though this was not significant (36). It remains difficult to understand the exact mechanisms of DAT expression, genotype, and the role of DAT in pathology, but the differences in DAT genotype and expression between healthy subjects and those who have disease might offer explanation of the crucial changes that reflect the pathologic mechanisms in disease. A larger study with randomly selected healthy subjects and striatal DAT availability measured with ${ }^{123} \mathrm{I}-\beta$ CIT is still needed, though, to confirm the effect of the VNTR; such a study could possibly replicate the association with the rs2652511-rs2937639-VNTR haplotype or find associations with other polymorphisms.

\section{CONCLUSION}

In this study, we examined the relationship between polymorphisms in the DAT gene and in vivo striatal DAT expression in 79 young adult Caucasian subjects. The 9R allele of the VNTR in the $3^{\prime}$ untranslated region of the DAT gene was associated with higher striatal DAT availability than was the $10 \mathrm{R}$ allele. Analysis suggested a dominant effect for the 9R allele. This finding supports the results of 2 previous studies on healthy subjects and striatal DAT binding. Two SNPs in the $5^{\prime}$ end of the gene, rs 2652511 and rs2937639, were not individually associated with striatal DAT availability. Haplotype analysis revealed that the identified effect of the $9 \mathrm{R}$ allele seems to be caused mainly by a subgroup of $9 \mathrm{R}$ carriers, the T-A-9R (rs2652511-
rs2937639-VNTR). These findings are valuable for further understanding of the mechanisms regulating DAT expression both in healthy people and in subjects with neuropsychiatric disorders.

\section{REFERENCES}

1. Thapar A, O'Donovan M, Owen MJ. The genetics of attention deficit hyperactivity disorder. Hum Mol Genet. 2005;14:R275-R282.

2. Drgon $\mathrm{T}$, Lin $\mathrm{Z}$, Wang $\mathrm{G}$, et al. Common human $5^{\prime}$ dopamine transporter (SLC6A3) haplotypes yield varying expression levels in vivo. Cell Mol Neurobiol. 2006;26:875-889.

3. Greenwood TA, Kelsoe JR. Promotor and intronic variants affect the transcriptional regulation of the human dopamine transporter gene. Genomics. 2003; $82: 511-519$

4. Michelhaugh SK, Fiskerstrand C, Lovejoy E, Bannon MJ, Quinn JP. The dopamine transporter gene (SLC6A3) variable number of tandem repeats domain enhances transcription in dopamine neurons. J Neurochem. 2001;79: 1033-1038.

5. Fuke S, Suo S, Takahashi N, Koike H, Sasagawa N, Ishiura S. The VNTR polymorphism of the human dopamine transporter (DAT1) gene affects gene expression. Pharmacogenomics J. 2001;1:152-156.

6. Miller GM, Madras BK. Polymorphisms in the 3-untranslated region of human and monkey dopamine transporter genes affect reporter gene expression. Mol Psychiatry. 2002;7:44-55.

7. Mill J, Asherson P, Craig I, D'Souza UM. Transient expression analysis of allelic variants of a VNTR in the dopamine transporter gene (DAT1). BMC Genet. 2005;6:3.

8. Jacobsen LK, Staley JK, Zoghbi SS, et al. Prediction of dopamine transporter binding availability by genotype: a preliminary report. Am J Psychiatry. 2000; 157:1700-1703.

9. van Dyck CH, Malison RT, Jacobsen LK, et al. Increased dopamine transporter availability associated with the 9-repeat allele of the SLC6A3 gene. J Nucl Med. 2005;46:745-751.

10. Heinz A, Goldman D, Jones DW, et al. Genotype influences in vivo dopamine transporter availability in human striatum. Neuropsychopharmacology. 2000; 22:133-139.

11. Cheon KA, Ryu YH, Kim JW, Cho DY. The homozygosity for 10-repeat allele at dopamine transporter gene and dopamine transporter density in Korean children with attention deficit hyperactivity disorder: relating to treatment response to methylphenidate. Eur Neuropsychopharmacol. 2005;15:95-101.

12. Martinez D, Gelernter J, Abi-Dargham A, et al. The variable number of tandem repeats polymorphism of the dopamine transporter gene is not associated with significant change in dopamine transporter phenotype in humans. Neuropsychopharmacology. 2001;24:553-560.

13. Lafuente A, Bernardo M, Mas S, et al. Dopamine transporter (DAT) genotype (VNTR) and phenotype in extrapyramidal symptoms induced by antipsychotics. Schizophr Res. 2007;90:115-122.

14. Lynch DR, Mozley PD, Sokol S, Maas NMC, Balcer LJ, Siderowf AD. Lack of effect of polymorphisms in dopamine metabolism related genes on imaging of TRODAT-1 in striatum of asymptomatic volunteers and patients with Parkinson's disease. Mov Disord. 2003;18:804-812.

15. Greenwood TA, Alexander M, Keck PE, et al. Segmental linkage disequilibrium within the dopamine transporter gene. Mol Psychiatry. 2002;7: $165-173$.

16. De Win MML, Jager G, Vervaeke H, et al. The Netherlands XTC Toxicity (NeXT) study: objectives and methods of a study investigating causality, course, and clinical relevance. Int J Methods Psychiatr Res. 2005;14:167-185.

17. De Win MML, Habraken JBA, Reneman L, Van den Brink W, Den Heeten GJ, Booij J. Validation of $\left[{ }^{123} \mathrm{I}\right]-\beta$-CIT SPECT to assess serotonin transporters in vivo in humans: a double-blind, placebo-controlled, crossover study with the selective serotonin reuptake inhibitor citalopram. Neuropsychopharmacology. 2005;30:996-1005.

18. Laruelle M, Wallace E, Seibyl JP, et al. Graphical, kinetic and equilibrium analysis of in vivo $\left[{ }^{123} \mathrm{I}\right] \beta$-CIT binding to dopamine transporters in healthy human subjects. J Cereb Blood Flow Metab. 1994;14:982-994.

19. Booij J, Tissingh G, Boer GJ, et al. [ $\left.{ }^{123} \mathrm{I}\right]$ FP-CIT SPECT shows a pronounced decline of striatal dopamine transporter labelling in early and advanced Parkinson's disease. J Neurol Neurosurg Psychiatry. 1997;62:133-140.

20. Innis RB, Cunningham VJ, Delforge J, et al. Concensus nomenclature for in vivo imaging of reversibly binding radioligands. J Cereb Blood Flow Metab. 2007;27: 1533-1539. 
21. Vandenbergh DJ, Persico AM, Hawkins AL, et al. Human dopamine transporter gene (DAT1) maps to chromosome 5p15.3 and displays a VNTR. Genomics. 1992;14:1104-1106.

22. Rozen S, Skaletsky HJ. Primer3 on the WWW for general users and for biologist programmers. In: Krawetz S, Misener S, eds. Bioinformatics Methods and Protocols: Methods in Molecular Biology. Totowa, NJ: Humana Press; 2000: 365-386.

23. van Dyck $\mathrm{CH}$, Seibyl JP, Malison RT, et al. Age-related decline in striatal dopamine transporter binding with iodine-123- $\beta$-CITSPECT. J Nucl Med. 1995;36:1175-1181.

24. Lavalaye J, Booij J, Reneman L, Habraken JBA, van Royen EA. Effect of age and gender on dopamine transporter imaging with [ $\left.{ }^{123} \mathrm{I}\right]-\mathrm{FP}-\mathrm{CIT}$ SPECT in healthy volunteers. Eur J Nucl Med. 2000;27:867-869.

25. Hosmer L, Lemeshow S. Applied Logistic Regression: Textbook and Solutions Manual. New York, NY: John Wiley \& Sons; 2001.

26. Tanck MWT, Klerkx AHEM, Jukema JW, de Knijff P, Kastelein JJP, Zwinderman AH. Estimation of multilocus haplotype effects using weighted penalised log-likelihood: analysis of five sequence variations at the cholesteryl ester transfer protein gene locus. Ann Hum Genet. 2003;67:175-184.

27. Purcell S, Cherny SS, Sham PC. Genetic power calculator: design of linkage and association genetic mapping studies of complex traits. Bioinformatics. 2003;19: $149-150$.

28. Staley JK, Krishnan-Sarin S, Zoghbi S, et al. Sex differences in [ $\left.{ }^{123} \mathrm{I}\right] \beta-\mathrm{CIT}$ SPECT measures of dopamine and serotonin transporter availability in healthy smokers and nonsmokers. Synapse. 2001;41:275-284.
29. Best SE, Sarrell PM, Malison RT, et al. Striatal dopamine transporter availability with $\left[{ }^{123} \mathrm{I}\right] \beta$-CIT SPECT is unrelated to gender or menstrual cycle. Psychopharmacology (Berl). 2005;183:181-189.

30. Reneman L, Booij J, Lavalaye J, et al. Use of amphetamine by recreational users of ecstasy (MDMA) is associated with reduced striatal dopamine transporter densities: a $\left[{ }^{123} \mathrm{I}\right]-\beta$-CIT SPECT study: preliminary report. Psychopharmacology (Berl). 2002;159:335-340.

31. Quickfall J, Crockford D. Brain neuroimaging in cannabis use: a review. J Neuropsychiatry Clin Neurosci. 2006;18:318-323.

32. Malison RT, Best SE, van Dyck $\mathrm{CH}$, et al. Elevated striatal dopamine transporters during acute cocaine abstinence as measured by $\left[{ }^{123} \mathrm{I}\right] \beta$-CIT SPECT. Am J Psychiatry. 1998;155:832-834.

33. Newberg A, Lerman C, Wintering N, Ploessl K, Mozley PD. Dopamine transporter binding in smokers and nonsmokers. Clin Nucl Med. 2007;32:452455.

34. de Win MML, Schilt T, Reneman L, et al. Ecstasy use and self-reported depression, impulsivity, and sensation seeking: a prospective cohort study. J Psychopharmacol. 2006;20:226-235.

35. Swanson JM, Kinsbourne M, Nigg J, et al. Etiologic subtypes of attentiondeficit/hyperactivity disorder: brain imaging, molecular genetic and environmental factors and the dopamine hypothesis. Neuropsychol Rev. 2007;17:39-59.

36. Kelada SNP, Checkoway H, Kardia SLR, et al. 5' and 3'region variability in the dopamine transporter gene (SLC6A3), pesticide exposure and Parkinson's disease risk: a hypothesis-generating study. Hum Mol Genet. 2006;15:30553062 . 